\title{
Endophenotypes of executive functions in obsessive compulsive disorder? A meta-analysis in unaffected relatives Eirini Zartaloudi ${ }^{\mathrm{a}}$, Keith R. Laws ${ }^{\mathrm{b}}$ and Elvira Bramon ${ }^{\mathrm{a}}$
}

\begin{abstract}
Endophenotypes are mediator traits between genetic influences and clinical phenotypes. Meta-analyses have consistently shown modest impairments of executive functioning in obsessive compulsive disorder (OCD) patients compared to healthy controls. Similar deficits have also been reported in unaffected relatives of OCD patients, but have not been quantified. We conducted the first metaanalysis combining all studies investigating executive functioning in unaffected relatives of individuals with OCD to quantify any deficits. A search of Pubmed, Medline and Psychlnfo databases identified 21 suitable papers comprising 707 unaffected relatives of OCD patients and 842 healthy controls. Effect sizes were calculated using random effects models. Unaffected relatives displayed a significant impairment in global executive functioning. Analyses of specific executive functioning subdomains revealed impairments in:
\end{abstract}

\section{Introduction}

Obsessive compulsive disorder (OCD) is a complex neuropsychiatric disorder with a lifetime prevalence of 2-3\% in the general population (Chamberlain et al., 2005). Despite several genetic loci being associated with OCD (Mattheisen et al., 2015; Arnold et al., 2018; Fernandez et al., 2018), its genetic architecture has not been fully deciphered yet and little is known about the mecha-nisms through which those genes lead to the disorder. The heterogeneity of symptomatology in OCD obscures the search for genetic mechanisms, leading to alterna-tive approaches. One such approach is the investigation of endophenotypes, which are heritable mediator traits between genetic influences and clinical phenotypes and are a risk factor for a disorder even if the person is not currently symptomatic (Gottesman and Shields, 1973; Gottesman et al., 1982; Gottesman and Gould, 2003). The rationale behind endophenotypes is that even if those traits are determined by multiple genes, their genetic architecture could be simpler than the clinically useful, but still based on diagnostic principles, psychiatric disor-der (Flint and Munafò, 2007; Lenzenweger, 2013).

Endophenotypic traits are intermediate measures of 'disease' between phenotype and genotype, which should be less genetically complex, be defined more

Supplemental Digital Content is available for this article. Direct URL citations appear in the printed text and are provided in the HTML and PDF versions of this article on the journal's website, www.psychgenetics.com. planning, visuospatial working memory and verbal fluency. Deficits in executive functioning are promising endophenotypes for OCD. To identify further biomarkers of disease risk/resilience in OCD, we suggest examining specific executive functioning domains. Psychiatr Genet 29: 211-219 Copyright (C) 2019 Wolters Kluwer Health, Inc. All rights reserved.

Keywords: cognitive functioning, endophenotype, executive functioning, meta-analysis, obsessive compulsive disorder

${ }^{a}$ Division of Psychiatry, University College London, London and ${ }^{\mathrm{b}}$ School of Life and Medical Sciences, University of Hertfordshire, Hertfordshire, UK

Correspondence to Eirini Zartaloudi, MSc, UCL Division of Psychiatry, 6th Floor Maple House, 149 Tottenham Court Road, London W1T 7NF, UK Tel: +44 207679 6562; e-mail: e.zartaloudi@ucl.ac.uk

straightforwardly than the actual disorder, resemble a physiological trait, and involve the same biochem-ical pathways but be closer to the level of gene action compared with the psychiatric disorder (Almasy and Blangero, 2001; Flint and Munafò, 2007; Glahn et al., 2014). Therefore, the relationship between genes and those traits should be stronger than with the disorder itself, because psychiatric disorders result from a combi-nation of genetic and nongenetic abnormalities impacted by environmental and sociocultural factors.

Several family studies have demonstrated that recurrence rates of neurocognitive endophenotypes in relatives of individuals with a psychiatric disorder are higher than prevalence in the general population, albeit the relatives do not exhibit the symptomatology of the psychiatric illness (Glahn et al., 2010; Drysdale et al., 2013; Kumar et al., 2015; Blakey et al., 2018). Research on neurocog-nitive endophenotypes had been partly led by evidence implicating structural and functional abnormalities in the frontal lobes (i.e., orbitofrontal cortex, anterior cingulate gyrus and the basal ganglia) and their links with the pathogenesis of OCD (e.g., for overviews, see Chamberlain et al., 2005; Piras et al., 2015). In particular, a key focus has been on executive function deficits as an endopheno-type of OCD and impairments have been found in OCD patients and, albeit to a lesser extent, in their unaffected relatives compared with healthy individuals (Maltby et al., 2005; Bannon et al., 2006; Cavedini et al., 2006; Lawrence et al., 2006; Olvet and Hajcak, 2008; Cavedini et al., 2010; 
Chamberlain and Menzies, 2009; Taylor et al., 2011; Mathews et al., 2012; Kashyap et al., 2013; Kloft et al., 2013).

Whether such executive deficits are a trait- or state-associated phenomenon has also received some attention in prepost treatment studies. It is controversial whether executive functioning deficits in OCD patients are sta-ble trait-like characteristics, or whether they might be state dependent, reflecting probable influences of the symptomatology on cognitive performance (Bannon et al., 2006). The findings on this issue are mixed in terms of whether neuropsychological deficits improve or not following symptomatic improvement (Abramovitch and Cooperman, 2015). Nevertheless, several studies have shown that executive deficits remain even in those whose OCD symptoms have remitted (Bannon et al., 2006; Rao et al., 2008; Sharma et al., 2014).

A meta-analysis of 115 studies with a total of 3452 OCD patients, reported that, compared with controls, OCD patients performed significantly worse in cognitive functioning in general and executive functioning in particular with a moderate effect size of $d=0.49$ for executive functioning (Abramovitch et al., 2013). Another meta-analysis of 88 published studies with a total of 3070 OCD patients and 3024 healthy controls reported that the patients perform significantly worse in all cognitive domains, including executive functioning (Shin et al., 2014). This cognitive impairment in patients was mild and the effect size for executive functioning in particular was $g=-0.49$. Authors highlighted how surprising that is, because exec-utive functioning is supposed to be the main affected domain in OCD patients. A more recent meta-analysis of 110 studies with a total of 3162 OCD patients and 3153 healthy controls, focussing solely on executive function-ing, reported that OCD patients were significantly more impaired in several domains including planning, inhibition, shifting and verbal fluency with effect sizes ranging from $d=0.3$ to 0.5 (Snyder et al., 2015).

The aim of this meta-analysis was to provide a quantitative evaluation of previously conducted research on executive functioning of unaffected relatives of patients with OCD and healthy subjects and also to check for moderator factors that may have affected the reported findings. Based on the majority of previous studies, it was expected that relatives would exhibit deficiencies in executive functioning compared to healthy controls. To the authors' knowledge, no previous meta-analysis of cognitive endophenotypes of OCD that includes unaffected relatives and healthy subjects has been conducted so far.

\section{Method}

\section{Search strategy and eligibility criteria}

A literature search was performed in Pubmed, Medline and PsychInfo databases, to identify papers investigating performance of unaffected relatives of OCD patients and unrelated healthy controls in executive functioning tasks. The search terms employed were: '(Obsessive Compulsive Disorder OR OCD) AND (cognit* OR execut* OR endophenot*)'. The time scale covered by our search was from January 2000 up to and including February 2019. A manual search was also performed in the reference list of the retrieved articles.

\section{Eligibility criteria}

The following criteria were set to assess eligibility for inclusion:

Inclusion criteria:

(1) Compare unaffected relatives of OCD patients with unrelated healthy controls.

(2) Include tasks measuring executive functioning.

(3) Report sufficient data to perform the statistical analyses. When means and SDs for the tasks of interest were not reported, we contacted the corresponding authors requesting additional information.

(4) Full text published article.

(5) English language restriction.

Exclusion criteria:

(1) Grey literature (articles not published in peer review journals).

(2) Studies reporting their samples are entirely included in other larger samples.

(3) Studies with nonhuman subjects.

(4) Meta-analytic studies.

(5) Systematic/literature reviews.

(6) Studies reporting only nonstandardized tasks.

The study selection procedure is illustrated graphically in the subsequent flowchart.

\section{Data extraction and analysis}

In the present meta-analysis, we compare unaffected rel-atives of OCD patients with healthy subjects. We did not compare OCD patients with healthy subjects because the meta-analyses by Shin et al. (2014), Abramovitch et al. (2013) and Snyder et al. (2015) have already quantified their differences and reported mild deficits in OCD patients.

The meta-analysis was conducted using the Comprehensive Meta-Analysis (CMA, version 3.3.070; Biostat, Englewood, New Jersey, USA). The executive function measures from each study included in the analysis are presented in Supplementary Table 1, Supplemental digital content 1, http://links.lww.com/PG/A229. The mean and SD from each task were extracted from each study. When these data were not available in a article, authors were contacted. Some studies reported multiple scores for each test. To account for that, we grouped the scores per test by study together to obtain one score per test. Then, we grouped the tests by each study together as a weighted average to produce one effect size per study. Some stud-ies were reporting the standard errors (SEs) from which we calculated the SDs. 
We calculated the Hedges' $g$ effect sizes, which are simi-lar to Cohen's $d$, but correct any potential bias that might result from small sample sizes (Hedges and Olkin, 2014). Heterogeneity among studies was assessed by examin-ing the distribution of effects sizes and calculating both Cochran's $Q$ and Higgin's $I^{2}$ statistics. Significant heterogeneity was found, indicating that differences across the effect sizes likely result from other sources than sampling error. Due to the moderate heterogeneity, the random effects model was employed. Meta-regression analyses for the mean age (measured in years), proportion of females and severity of illness [measured by the Yale-Brown Obsessive Compulsive Scale (Y-BOCS)] were performed.

The tasks we included in the meta-analysis are: Cambridge Gambling task (Rogers et al., 1999), Iowa Gambling Task (Bechara et al., 1994), Game of Dice task (Brand et al., 2005), Flanker task (Eriksen and Eriksen, 1974), Stroop task (Wechsler, 1991), Stop signal task (Aron et al., 2004), Digit Vigilance Test (Lezak et al., 2004), Tower of London (TOL; Shallice, 1982), Tower of Hanoi (ToH; Welsh et al., 2000), One touch spatial planning task (Williams-Gray, 2007), Wisconsin Card Sorting Test (Milner, 1963), Intradimensional/Extradimensional Shift task (Cambridge Cognition, 1996), Trail Making Test Part B (Reitan, 1995), Digit Span Backward (Wechsler, 1991), Figural memory test (Endicott et al., 1976), Visual organ-ization test (Hooper, 1958), Delayed Alternation Test (Freedman, 1990), Design Fluency task (Benton, 1968), Situational Awareness test (Endsley, 1995), Wechsler Memory Scale (Wechsler, 1991), Visual Memory sub-test (Wechsler, 1991), Visual working memory test (Hooper, 1958), Verbal Fluency test (Jones-Gotman and Milner, 1977), Controlled Oral Word Association Test (Bechtoldt et al., 1962), Controlled Word Association Test (Wechsler, 1991), Category Fluency test (Wechsler, 1991), Association fluency task (Delorme et al., 2017) and Verbal Fluency task (Lezak et al., 2004).

Owing to the high heterogeneity between executive functions, we analysed each specific executive func-tioning domain separately. Six domains were defined: Planning, Inhibition-Selective Attention, Set-shifting, Decisionmaking, Visuospatial Working Memory and Verbal Fluency. Each task was categorized to one of the aforementioned domains and the tasks that were grouped together were then combined as a weighted average to obtain effect sizes for all studies in each domain. Eventually, we performed an analysis evaluating global executive functioning by grouping all the domains together.

\section{Results}

The number of articles yielded by the systematic review was 12716 and the Preferred Reporting Items for Systematic Reviews and Meta-Analyses guide-lines were employed to identify the articles of interest.
An additional article was identified by hand search. Initially, 8299 duplicates were excluded. The titles and abstracts of the 4418 remaining articles were screened and 3554 articles were excluded for being extrane-ous, resulting in 864 articles. The retrieved set of arti-cles was screened in relation to the eligibility criteria. Eventually, 21 studies were deemed relevant and were therefore included in the meta-analysis (for additional information regarding those articles, see Supplementary Table 1, Supplemental digital content 1, http://links.lww. com/PG/A229). Quality assessment tool for diagnostic accuracy studies was employed to assess study quality (Whiting et al., 2004).

\section{Demographics}

Demographic characteristics of the unaffected first-de-gree relatives $(n=707)$ and healthy controls $(n=842)$ are shown in Table 1.

\section{Analyses of executive functioning domains}

Executive functions can cover a range of different cognitive processes, and we grouped together tests measur-ing specific executive functioning domains. Table 2 lists the domains of executive functioning for which we con-ducted additional analyses, the number of studies pro-viding data and the tasks for each domain along with the number of participants.

Initially, differences were observed in inhibition/selec-tive attention, visuospatial working memory, verbal fluency and planning, with unaffected relatives under-performing in all domains compared with healthy con-trols. After adjusting for multiple comparisons, by dividing the threshold of significance by the number of comparisons $(P=0.008)$ significant differences remained in the domains of visuospatial working memory, verbal fluency and planning with small-to-moderate effect sizes. The effect sizes for each comparison are summarized in Table 3.

\section{Comparison of unaffected first-degree relatives versus healthy controls in global executive functioning}

When compared with controls, we found significant executive function impairments among the unaffected relatives of people with OCD. The pooled standardized difference in means between the unaffected relatives and control groups (with the 21 eligible studies included) was Hedge's $g=0.25$ and was highly significant $(P<0.001)$. We systematically assessed the heterogeneity among studies which was found to be high $(Q=66.41, \mathrm{df}=20$, $\left.P<0.001, I^{2}=69.88\right)$. Therefore, the random effects model is reported. The forest plot demonstrating the differences between the unaffected relatives and healthy controls is presented in Fig. 2.

Funnel asymmetry was assessed with Egger's test (0. 66) and showed no evidence of publication bias in the results $(P=0.51)$. A funnel plot illustrating the 
Table 1 Demographic characteristics of the unaffected first-degree relatives $(n=707)$ and healthy controls $(n=842)$

\begin{tabular}{|c|c|c|c|c|c|c|c|}
\hline & \multicolumn{3}{|c|}{ Unaffected relatives } & \multicolumn{4}{|c|}{ Healthy controls } \\
\hline & Female \% & Age (SD) & Age range & Y-BOCS & Female \% & Age (SD) & Age range \\
\hline Carrasco et al. (2013) & 31.6 & $13.9(2.4)$ & $10-17$ & - & 50.0 & $13.8(2.3)$ & $10-17$ \\
\hline Zhang et al. (2015a) & 50.0 & $25.3(6.4)$ & $18-40$ & - & 47.5 & $25.0(5.9)$ & $18-40$ \\
\hline Zhang et al. (2015b) & 52.7 & $28.4(7.3)$ & - & 1.9 & 56.4 & $27.9(7.3)$ & - \\
\hline Lochner et al. (2016) & 84.6 & $46.9(5.3)$ & $18-59$ & - & 59.3 & $31.4(11.1)$ & $18-59$ \\
\hline Li et al. (2012) & - & - & - & - & 45.0 & $22.6(5.8)$ & - \\
\hline Rajender et al. (2011) & 60.0 & $26.4(3.9)$ & - & - & 60.0 & $26.9(2.9)$ & - \\
\hline Lennertz et al. (2012) & 60.0 & $42.1(14.3)$ & $18-65^{\mathrm{a}}$ & 0.1 & 70.0 & $42.7(12.6)$ & $18-65^{\mathrm{a}}$ \\
\hline Bey et al. (2018) & 78.4 & $48.8(12.5)$ & - & - & 57.8 & $34.1(12.0)$ & - \\
\hline Chamberlain et al. (2007) & 65.0 & $34.2(11.4)$ & - & 3.7 & 65.0 & $33.1(10.5)$ & - \\
\hline $\begin{array}{l}\text { Riesel et al. (2011) } \\
\text { Segalàs et al. }(2010)\end{array}$ & 56.7 & $45.9(13.0)$ & $18-65^{\mathrm{a}}$ & - & $\begin{array}{l}56.7 \\
52.0\end{array}$ & $45.4(12.8)$ & ${ }_{-}^{18-65^{a}}$ \\
\hline Cavedini et al. (2010) & 40.1 & $45.0(17.7)$ & $18-65^{a}$ & - & 71.0 & $34.7(16.1)$ & $18-65^{a}$ \\
\hline de Wit et al. (2012) & 29.0 & $38.3(13.4)$ & - & 0.1 & 51.0 & $39.7(11.6)$ & - \\
\hline Menzies et al. (2007) & 70.9 & $36.7(13.4)$ & - & 1.7 & 64.6 & $33.4(11.1)$ & - \\
\hline Ozcan et al. (2016) & 38.9 & $31.8(11.5)$ & $18-65^{\mathrm{a}}$ & - & 42.9 & $32.4(8.0)$ & $18-65^{\mathrm{a}}$ \\
\hline Riesel et al. (2019) & 64 & 45 (14.8) & - & - & 58 & $32.1(9.9)$ & - \\
\hline Vaghi et al. (2017) & 73.7 & $41.1(10.6)$ & - & - & 75.0 & $36.4(8.5)$ & - \\
\hline van Velzen et al. (2015) & 33.3 & $38.3(13.4)$ & - & - & 40.0 & $38.2(11.6)$ & - \\
\hline Delorme et al. (2007) & 45.0 & $42.3(15.0)$ & - & - & 45.0 & $39.7(18.2)$ & - \\
\hline Tezcan and Tümkaya (2018) & 62 & $37.9(15.3)$ & $18-65^{\mathrm{a}}$ & - & 68.3 & $35.8(12.5)$ & $18-65^{\mathrm{a}}$ \\
\hline Viswanath et al. (2009) & 40.0 & $27.5(6.9)$ & $18-45^{\mathrm{a}}$ & - & 40.0 & $27.4(6.4)$ & $18-45$ \\
\hline
\end{tabular}

Y-BOCS, Yale-Brown Obsessive Compulsive Scale.

${ }^{a}$ Studies only report the age ranges for eligibility in the study.

Table 2 The executive function domains tested, the tasks measuring each function and the number of studies

\begin{tabular}{|c|c|c|c|c|c|}
\hline \multirow[b]{2}{*}{ Executive functioning domain } & \multicolumn{2}{|c|}{ Number of participants } & \multirow[b]{2}{*}{ Tasks } & \multicolumn{2}{|c|}{ Number of participants } \\
\hline & FDRs & Controls & & FDRs & Controls \\
\hline \multirow[t]{4}{*}{ Inhibition - selective attention $(k=12)$} & 347 & 479 & Stop signal task $(k=4)$ & 80 & 108 \\
\hline & & & Stroop task $(k=5)$ & 168 & 171 \\
\hline & & & Digit vigilance test $(k=1)$ & 30 & 30 \\
\hline & & & Flanker task $(k=3)$ & 99 & 200 \\
\hline \multirow[t]{6}{*}{ Verbal fluency $(k=8)$} & 378 & 359 & Controlled word association Test $(k=1)$ & 18 & 21 \\
\hline & & & Category fluency test $(k=1)$ & 18 & 21 \\
\hline & & & Verbal fluency task $(k=4)$ & 197 & 172 \\
\hline & & & Digit span backward $(k=4)$ & 138 & 141 \\
\hline & & & Association fluency task $(k=1)$ & 64 & 47 \\
\hline & & & Controlled oral word association test $(k=1)$ & 25 & 25 \\
\hline \multirow[t]{3}{*}{ Set-shifting $(k=10)$} & 365 & 339 & Wisconsin card sorting test $(k=7)$ & 251 & 242 \\
\hline & & & Trail making test part $\mathrm{B}(k=7)$ & 280 & 258 \\
\hline & & & Intradimensional/extradimensional shift task $(k=1)$ & 20 & 20 \\
\hline \multirow[t]{3}{*}{ Decision making $(k=5)$} & 148 & 158 & Cambridge gambling task $(k=2)$ & 33 & 47 \\
\hline & & & lowa gambling task $(k=3)$ & 115 & 111 \\
\hline & & & Game of dice task $(k=1)$ & 55 & 55 \\
\hline \multirow[t]{6}{*}{ Visuospatial working memory $(k=5)$} & 171 & 157 & Visual working memory test $(k=1)$ & 30 & 30 \\
\hline & & & Figural memory test $(k=1)$ & 18 & 21 \\
\hline & & & Visual organization test $(k=1)$ & 30 & 30 \\
\hline & & & Wechsler memory scale visual memory subtest $(k=1)$ & 48 & 40 \\
\hline & & & Situational awareness test $(k=1)$ & 50 & 41 \\
\hline & & & Delayed alternation test $(k=1)$ & 25 & 25 \\
\hline \multirow[t]{4}{*}{ Planning $(k=10)$} & 486 & 467 & Tower of Hanoi $(k=3)$ & 123 & 111 \\
\hline & & & Tower of London $(k=6)$ & 280 & 289 \\
\hline & & & Design fluency task $(k=1)$ & 64 & 47 \\
\hline & & & One touch spatial planning task $(k=1)$ & 47 & 20 \\
\hline
\end{tabular}

FDRs, unaffected first degree relatives.

distribution of studies around the combined effect size was produced (see Supplementary Fig. 7, Supplemental digital content 1, http://links.lww.com/PG/A229). The studies were distributed symmetrically around the combined effect size, without a higher concentration of studies on either side. Therefore, we can safely report no publication bias.

\section{Moderator analyses}

Given the heterogeneity across studies, metaregression analyses were conducted to investigate whether additional characteristics contributed to the variation between study effect sizes. Mean age, proportion of females and severity of patient OCD as measured by the Y-BOCS scale in each study were examined as moderators. Only age was found 
Table 3 Statistics for each executive functioning domain

\begin{tabular}{|c|c|c|c|c|c|}
\hline & Hedge's $g$ & Lower $95 \% \mathrm{Cl}$ & Upper $95 \% \mathrm{Cl}$ & $P$ value & $Q$ \\
\hline Inhibition/selective attention & 0.27 & 0.05 & 0.49 & 0.016 & $52.81(\mathrm{df}=11, P<0.001)$ \\
\hline Set-shifting & 0.11 & -0.05 & 0.28 & 0.19 & $18.57(\mathrm{df}=9, P=0.029)$ \\
\hline Decision-making & 0.45 & 0.04 & 0.86 & 0.03 & $35.53(\mathrm{df}=4, P<0.001)$ \\
\hline Visuospatial working memory & 0.36 & 0.19 & 0.53 & $<0.001$ & $7.20(\mathrm{df}=4, P=0.126)$ \\
\hline Verbal fluency & 0.20 & 0.09 & 0.32 & $<0.001$ & $5.09(\mathrm{df}=7, P=0.65)$ \\
\hline Planning & 0.37 & 0.19 & 0.56 & $<0.001$ & $23.44(\mathrm{df}=9, P=0.003)$ \\
\hline
\end{tabular}

Forest plots for each comparison are included in the supplementary material (Supplementary Figs. 1-6, Supplemental digital content 1, http://links.Iww.com/PG/A229). $\mathrm{Cl}$, confidence interval.

Fig. 1

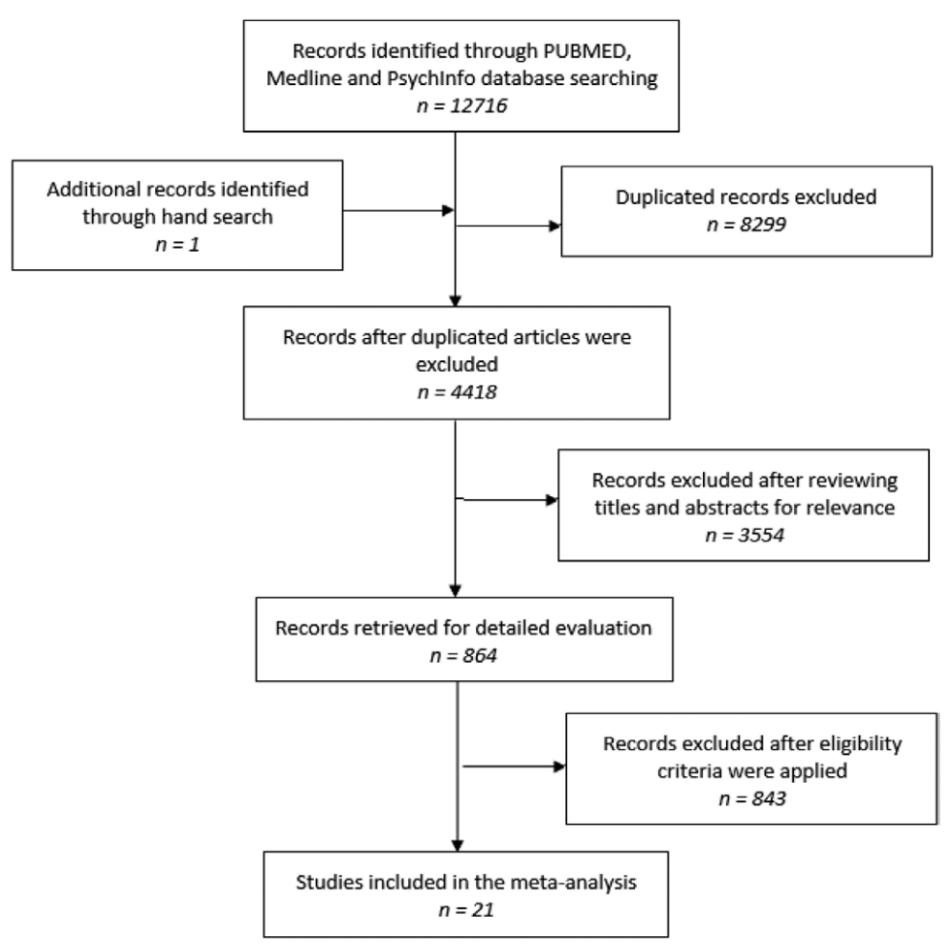

Preferred Reporting Items for Systematic Reviews and Meta-Analyses (2009) flow diagram illustrating the study selection procedure.

to be a significant moderator with relatives with higher average age exhibiting more deficits (Table 4).

\section{Sensitivity analyses}

Sensitivity analyses uncovered no outliers and confirmed the robustness of our results.

\section{Discussion}

The purpose of this meta-analysis was to provide a quantitative assessment of research comparing executive functioning in unaffected relatives of patients with OCD and healthy individuals. Our meta-analysis included 21 primary studies with 1549 subjects (707 unaffected relatives and 842 healthy controls). Our analysis revealed a small ( $g=0.25)$, but significant overall executive function impairment in the unaffected first-degree relatives of those diagnosed with OCD.

Compared with healthy controls, the unaffected relatives of patients with OCD showed evidence of impairments in global executive functioning. Previous meta-analyses 
Fig. 2

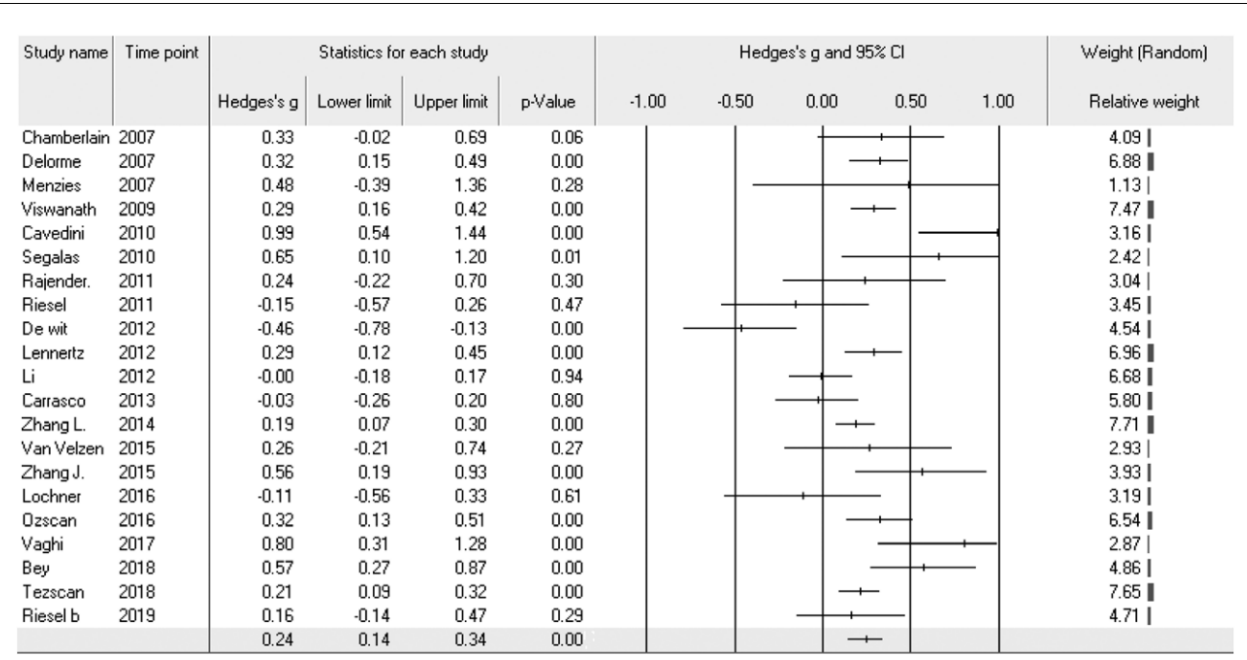

Forest plot of the meta-analysis of all 21 eligible primary studies comparing unaffected relatives of patients with OCD and unrelated healthy con-trols. Positive effect sizes favour healthy controls, whereas negative effect sizes favour unaffected relatives. OCD, .

Table 4 Statistics for the moderator analyses for age, proportion of females and severity of illness

\begin{tabular}{lccccrrr}
\hline & $Q$ & $\beta$ & $S E$ & $Z$ & Lower Cl & Upper Cl & $P$ value \\
\hline Age $(k=20)$ & $9.11(P=0.002)$ & 0.013 & 0.004 & 3.02 & 0.005 & 0.022 \\
Female proportion $(k=20)$ & $3.41(P=0.061)$ & 0.007 & 0.004 & 1.85 & -0.001 & 0.002 \\
Severity of illness $(k=5)$ & $1.46(P=0.002)$ & 0.005 & 0.001 & 0.68 & -0.011 & 0.016 & 0.064 \\
\hline
\end{tabular}

$\mathrm{Cl}$, confidence interval.

comparing people with OCD and healthy controls have consistently reported mild-to-moderate severity execu-tive function deficits $(d=0.49$ : Abramovitch et al., 2013; $g=-0.49$ : Shin et al., 2014; $d=0.3$ to 0.5: Snyder et al., 2015), and the evidence presented here suggests that such deficits extend to their unaffected relatives - albeit at a smaller level. Interestingly, the two meta-analyses that assessed overall executive function in OCD patients, both found effect sizes (Shin et al., 2014: $g=-0.49$, 95\% CI, -0.55 to -0.43 ; Abramovitch et al 2013: $-0.498,95 \%$ CI, -0.58 to -0.42 ) approximately twice that reported here for OCD relatives and notably the $95 \%$ CIs did not over-lap between OCD patients and OCD relatives reported here. A crucial criterion for an endophenotype is that it must be impaired in people with genetic predisposition for the disease and our data indicate this criterion is met.

In the analyses of specific domains of executive functioning, unaffected relatives significantly underperformed in visuospatial working memory, verbal fluency and planning with subtle to moderate deficits. We found no differences between unaffected relatives and controls in inhibition/ selective attention, decision-making and set-shifting. Indeed, of the 10 studies examining set-shifting, only one (Cavedini et al., 2010) reported a significant impairment in OCD relatives. This appears to accord with Shin et al. (2014) meta-analysis across a range of executive tasks in OCD patients. They found a wide range of performance across executive areas with setshifting being among the smallest effect sizes and nonsignificant $(-0.31$ for extra-dimensional shifting), while planning $(\mathrm{ToL}, \mathrm{ToH})$ the largest $(-0.73)$. Regarding decisionmaking, there were only five primary studies and power was probably limited for this domain.

Turning to moderators of executive impairment in the relatives of people with $\mathrm{OCD}$, neither the proportion of females in each study nor severity of symptoms in the unaffected relatives (as measured by the Y-BOCS scale) were significant moderators of effect size. In line with the three meta-analyses comparing OCD patients with 
healthy controls, we also found that illness severity was not a significant moderator of cognitive performance. By contrast, age was associated with executive impairment, with increasing age of relatives being associated with greater executive impairment. The three meta-analyses that have assessed executive function in OCD patients are inconsistent on the role of age - with findings that executive impairment and age were unrelated (Abramovitch et al., 2013), positively related (Snyder et al., 2015) and negatively related (Shin et al., 2014). In line with the findings for OCD relatives reported here, Snyder et al (2015) found that deficits were greater for older OCD samples (for set-shifting, visuospatial WM, verbal fluency and planning) and suggest that further research is required to examine the relationship, especially as age has not been a focus in the primary studies.

The tasks that are frequently used to assess executive functions are of course complex and multifactorial. The tests also often involve nonexecutive function abilities, making the interpretation of findings rather challenging (Miyake et al., 2000; Aron, 2008). Additionally, some tasks are viewed as executive (in nature) by some researchers, but not others. For example, inconsistencies exist in the literature concerning Rey's Complex Figure Test (RCFT), with some arguing that aside from assessing visuocon-structional ability, it measures executive functioning, specifically the domains of planning and organisation (Somerville et al., 2000; Watanabe et al., 2005). Others, how-ever, suggest the Rey Figure focuses almost exclusively on visuoperceptual and visuocontructional skills (Beebe et al, 2004; Schwarz et al., 2009). For the present meta-analy-sis, we therefore chose not to include tasks such as RCFT and the Complex Figure Test (CFT), which might be con-tentious. Future research should employ tasks measuring specifically executive functioning to avoid this issue.

Although we did not find symptomatology to be a predictor of effect size, this is perhaps unsurprising given that few studies reported Y- BOCS scores and because the levels were close to floor in relatives. Nevertheless, symptomatology of OCD patients themselves is vastly heterogeneous with each symptom potentially impact-ing an individuals' daily and cognitive functioning in a different manner. By way of example, patients with intrusive thoughts might perform worse in executive tasks owing to lack of concentration and depletion of cognitive resources, compared with patients with order-liness obsession. It has also been reported that OCD patients with hoarding symptoms exhibit relatively dif-ferent neuropsychological deficits than OCD patients without hoarding (Tolin et al., 2011). Studies typically do not report information regarding the specific symp-tomatology of their participants, which could potentially be an important moderator to examine. We would there-fore encourage empirical research aimed at recruiting patients with homogeneous symptomatology and report further information on the symptoms their participants exhibit.

Of course, poor performance on executive tasks by OCD patients may be confounded by a variety of factors, including symptomatology and medication. In the current meta-analysis, unaffected relatives were, of course, relatively symptom-free and not treated with psychotropic medication, Thus, the deficits reported here cannot be attributed to potential treatment confounds. Because less severe version of the same executive dysfunction occurs in healthy relatives is also consistent with the notion that the deficit in patients may be primary rather than second-ary confounds.

The current meta-analysis contains several strengths. As far as we are aware, this is the first meta-analysis quanti-fying how unaffected relatives of OCD patients perform in executive functioning tasks compared with healthy unrelated individuals and the potential of executive functioning performance as disease endophenotype. It also investigates potential moderators and specific domains of executive functioning. All included studies employed the Y-BOCS scale to measure severity of OCD symptomatology, which ensured consistency. Furthermore, we found no evidence of publication bias. The limitations of this metaanalysis should be noted. In regards to the executive function domain analyses, there are not reliable criteria to classify each cognitive test under a specific domain; therefore, we did so based on existing psychometric evidence.

\section{Conclusion}

In conclusion, this meta-analysis shows that the unaf-fected relatives of people with OCD have global exec-utive function impairments and show mild-to-moderate deficits in several executive function domain analyses. These deficits have also been reported among patients with OCD and could therefore be endophenotypic mark-ers of genetic predisposition. Therefore, our meta-analy-sis indicates that it is vital to examine specific cognitive subdomains in the quest for endophenotypes and bio-markers of disease risk/resilience.

Our findings indicate that the deficits in executive functioning, which have been quantified by three pre-vious large meta-analyses comparing OCD patients with healthy subjects (Abramovitch et al., 2013; Shin et al., 2014; Snyder et al., 2015) seem to be an endopheno-type of OCD, because they do extend to the unaffected relatives of the patients. When we focussed on specific domains, deficits in visuospatial working memory, ver-bal fluency and planning were also found in unaffected relatives, suggesting that some quite specific aspects of executive dysfunction have endophenotypic qualities. To substantiate our conclusion, we encourage future empirical research to focus on specific executive func-tion domains instead of grouping the domains together, so as to identify any potential endophenotypes of OCD. 


\section{Acknowledgements}

Dr Bramon acknowledges the following support: BMA Margaret Temple grants 2016 and 2006, MRC Korean Health Industry Development Institute Partnering Award (MC_ PC_16014), MRC New Investigator Award and a MRC Centenary Award (G0901310), National Institute of Health Research UK postdoctoral fellow-ship, the Psychiatry Research Trust, the Schizophrenia Research Fund, the Brain and Behaviour Research foundation's NARSAD Young Investigator Awards 2005, 2008, Wellcome Trust Research Training Fellowship, the NIHR Biomedical Research Centre for Mental Health at the South London and Maudsley NHS Foundation Trust and Institute of Psychiatry Kings College London and the NIHR Biomedical Research centre at UCL.

This study was supported by the NIHR Biomedical Research Centre at University College London (mental health theme).

All authors have made significant contributions to this manuscript in one or more of the following areas: design of the study, data analysis and interpretation, drafting and revising the manuscript. All authors have given final approval of this manuscript.

\section{Conflicts of interest}

There are no conflicts of interest.

\section{References}

Abramovitch A, Abramowitz JS, Mittelman A (2013). The neuropsychology of adult obsessive-compulsive disorder: a meta-analysis. Clin Psychol Rev 33:1163-1171.

Abramovitch A, Cooperman A (2015). The cognitive neuropsychology of obses-sive-compulsive disorder: A critical review. J Obsessive Compuls Relat Disord, 5:24-36.

Almasy L, Blangero J (2001). Endophenotypes as quantitative risk factors for psychiatric disease: rationale and study design. Am J Med Genet 105:42-44.

Arnold PD, Askland KD, Barlassina C, Bellodi L, Bienvenu OJ, Black D, et al. (2018). Revealing the complex genetic architecture of obsessivecompulsive disorder using meta-analysis. Mol Psychiatry 23:1181-1188.

Aron AR (2008). Progress in executive-function research. Curr Dir Psychol Sci 17:124-129.

Aron AR, Robbins TW, Poldrack RA (2004). Inhibition and the right inferior frontal cortex. Trends Cogn Sci 8:170-177.

Bannon S, Gonsalvez CJ, Croft RJ, Boyce PM (2006). Executive functions in obsessive-compulsive disorder: state or trait deficits? Aust $N Z \mathrm{~J}$ Psychiatry 40:1031-1038.

Bechara A, Damasio AR, Damasio H, Anderson SW (1994). Insensitivity to future consequences following damage to human prefrontal cortex. Cognition 50:7-15.

Bechtoldt HP, Benton AL, Fogel ML (1962). An application of factor analysis in neuropsychology. Psychological Rec 12:147.

Beebe DW, Ris MD, Brown TM, Dietrich KN (2004). Executive functioning and memory for the rey-osterreith complex figure task among community adoles-cents. Appl Neuropsychol 11:91-98.

Benton AL (1968). Differential behavioral effects in frontal lobe disease. Neuropsychologia 6:53-60.

Bey K, Kaufmann C, Lennertz L, Riesel A, Klawohn J, Heinzel S, et al. (2018). Impaired planning in patients with obsessive-compulsive disorder and unaf-fected first-degree relatives: evidence for a cognitive endophenotype. J Anxiety Disord 57:24-30.

Blakey R, Ranlund S, Zartaloudi E, Cahn W, Calafato S, Colizzi M, et al.; GROUP (2018). Associations between psychosis endophenotypes across brain functional, structural, and cognitive domains. Psychol Med 48:1325-1340.
Brand M, Fujiwara E, Borsutzky S, Kalbe E, Kessler J, Markowitsch HJ (2005). Decisionmaking deficits of Korsakoff patients in a new gambling task with explicit rules: associations with executive functions. Neuropsychology 19:267-277.

Cambridge Cognition (1996). CANTAB. Cambridge: Cambridge Cognition Ltd. Carrasco M, Harbin SM, Nienhuis JK, Fitzgerald KD, Gehring WJ, Hanna GL (2013). Increased error-related brain activity in youth with obsessive-compulsive disorder and unaffected siblings. Depress Anxiety 30:39-46.

Cavedini P, Gorini A, Bellodi L (2006). Understanding obsessive-compulsive dis-order: focus on decision making. Neuropsychol Rev 16:3-15.

Cavedini P, Zorzi C, Piccinni M, Cavallini MC, Bellodi L (2010). Executive dysfunctions in obsessive-compulsive patients and unaffected relatives: searching for a new intermediate phenotype. Biol Psychiatry 67:1178-1184.

Chamberlain SR, Menzies L (2009). Endophenotypes of obsessivecompulsive disorder: rationale, evidence and future potential. Expert Rev Neurother 9:1133-1146.

Chamberlain SR, Blackwell AD, Fineberg NA, Robbins TW, Sahakian BJ (2005). The neuropsychology of obsessive compulsive disorder: the importance of failures in cognitive and behavioural inhibition as candidate endophenotypic markers. Neurosci Biobehav Rev 29:399-419.

Chamberlain SR, Fineberg NA, Menzies LA, Blackwell AD, Bullmore ET, Robbins TW, et al. (2007). Impaired cognitive flexibility and motor inhibition in unaf-fected first-degree relatives of patients with obsessivecompulsive disorder. Am J Psychiatry, 164:335-338.

de Wit SJ, de Vries FE, van der Werf YD, Cath DC, Heslenfeld DJ, Veltman $E M$, et al. (2012). Presupplementary motor area hyperactivity during response inhi-bition: a candidate endophenotype of obsessivecompulsive disorder. Am J Psychiatry 169:1100-1108.

Delorme R, Goussé V, Roy I, Trandafir A, Mathieu F, Mouren-Siméoni MC, et al. (2007). Shared executive dysfunctions in unaffected relatives of patients with autism and obsessive-compulsive disorder. Eur Psychiatry 22:32-38.

Drysdale E, Knight HM, McIntosh AM, Blackwood DH (2013). Cognitive endophenotypes in a family with bipolar disorder with a risk locus on chromosome 4. Bipolar Disord 15:215-222.

Endicott J, Spitzer RL, Fleiss JL, Cohen J (1976). The global assessment scale. A procedure for measuring overall severity of psychiatric disturbance. Arch Gen Psychiatry 33:766-771.

Endsley MR (1995). Measurement of situation awareness in dynamic systems. Human factors 37:65-84.

Eriksen BA, Eriksen CW (1974). Effects of noise letters upon the identification of a target letter in a nonsearch task. Percept psychophys 16:143-149.

Fernandez TV, Leckman JF, Pittenger C (2018). Genetic susceptibility in obses-sive-compulsive disorder. Handb Clin Neurol 148:767-781.

Flint J, Munafò MR (2007). The endophenotype concept in psychiatric genetics. Psychol Med 37:163-180

Freedman M (1990). Object alternation and orbitofrontal system dysfunction in alzheimer's and parkinson's disease. Brain Cogn 14:134-143.

Glahn DC, Almasy L, Barguil M, Hare E, Peralta JM, Kent J W Jr, et al. (2010). Neurocognitive endophenotypes for bipolar disorder identified in multiplex multigenerational families. Arch Gen Psychiatry 67:168-177.

Glahn DC, Knowles EE, McKay DR, Sprooten E, Raventós H, Blangero J, et al. (2014). Arguments for the sake of endophenotypes: examining common misconceptions about the use of endophenotypes in psychiatric genetics. $A m \mathrm{~J}$ Med Genet B Neuropsychiatr Genet 165B:122-130.

Gottesman II, Gould TD (2003). The endophenotype concept in psychiatry: ety-mology and strategic intentions. Am J Psychiatry 160:636-645.

Gottesman II, Shields J (1973). Genetic theorizing and schizophrenia. $\mathrm{Br} \mathrm{J}$ Psychiatry 122:15-30.

Gottesman I, Shields J, Hanson DR (1982). Schizophrenia, The Epigenetic Puzzle. Cambridge: Cambridge University Press.

Hedges L, Olkin I (2014). Statistical Methods For Meta-Analysis. Orlando, Florida: Academic press.

Hooper HE (1958). The Hooper visual Organization Test. Los Angeles: Western Psychological Services.

Jones-Gotman M, Milner B (1977). Design fluency: the invention of nonsense drawings after focal cortical lesions. Neuropsychologia 15:653-674.

Kashyap H, Kumar JK, Kandavel T, Reddy YC (2013). Neuropsychological func-tioning in obsessive-compulsive disorder: are executive functions the key defi-cit? Compr Psychiatry 54:533-540.

Kloft L, Reuter B, Riesel A, Kathmann N (2013). Impaired volitional saccade con-trol: first evidence for a new candidate endophenotype in obsessivecompul-sive disorder. Eur Arch Psychiatry Clin Neurosci 263:215-222.

Kumar A, Solanki R, Satija Y, Gupta S, Singh P (2015). Neurocognitive endo-phenotypes in first degree relatives of bipolar affective disorder: an Indian experience. Eur Psychiatry 30:179.

Lawrence NS, Wooderson S, Mataix-Cols D, David R, Speckens A, Phillips $\mathrm{ML}$ (2006). Decision making and set shifting impairments are 
associated with distinct symptom dimensions in obsessive-compulsive disor-der. Neuropsychology 20:409-419.

Lennertz L, Rampacher F, Vogeley A, Schulze-Rauschenbach S, Pukrop R, Ruhrmann S, et al. (2012). Antisaccade performance in patients with obsessive-compulsive disorder and unaffected relatives: further evidence for impaired response inhibition as a candidate endophenotype. Eur Arch Psychiatry Clin Neurosci 262:625-634.

Lenzenweger MF (2013). Endophenotype, intermediate phenotype, biomarker: definitions, concept comparisons, clarifications. Depress Anxiety 30:185-189.

Lezak MD, Howieson DB, Loring DW, Fischer JS (2004). Neuropsychological Assessment. USA: Oxford University Press.

Li B, Sun JH, Li T, Yang YC (2012). Neuropsychological study of patients with obsessive-compulsive disorder and their parents in China: searching for potential endophenotypes. Neurosci Bull 28:475-482.

Lochner C, Chamberlain SR, Kidd M, Fineberg NA, Stein DJ (2016). Altered cognitive response to serotonin challenge as a candidate endophenotype for obsessive-compulsive disorder. Psychopharmacology (Berl) 233: 883-891.

Maltby N, Tolin D F, Worhunsky P, O'Keefe TM, Kiehl KA (2005). Dysfunctional action monitoring hyperactivates frontal-striatal circuits in obsessive-compulsive disorder: an Event-Related Fmri Study. Neuroimage 24:495-503.

Mathews CA, Perez VB, Delucchi KL, Mathalon DH (2012). Error-related nega-tivity in individuals with obsessive-compulsive symptoms: toward an under-standing of hoarding behaviors. Biol Psychol 89:487-494.

Mattheisen M, Samuels JF, Wang Y, Greenberg BD, Fyer AJ, McCracken JT, et al. (2015). Genome-wide association study In obsessive-compulsive disorder: results from the ocgas. Mol Psychiatry 20:337-344.

Menzies L, Achard S, Chamberlain SR, Fineberg N, Chen CH, del Campo N, et al. (2007). Neurocognitive endophenotypes of obsessive-compulsive disorder. Brain 130:3223-3236.

Milner B (1963). Effects of different brain lesions on card sorting: the role of the frontal lobes. Arch Neurol 9:90-100.

Miyake A, Friedman NP, Emerson MJ, Witzki AH, Howerter A, Wager TD (2000). The unity and diversity of executive functions and their contributions to com-plex 'frontal lobe' tasks: a latent variable analysis. Cogn Psychol 41:49-100. New Jersey, Englewood. (2014). Comprehensive Meta-Analysis Software (CMA) (Version2)

Olvet DM, Hajcak G (2008). The error-related negativity (ERN) and psychopathology: toward an endophenotype. Clin Psychol Rev 28:1343-1354.

Ozcan H, Ozer S, Yagcioglu S (2016). Neuropsychological, electrophysiological and neurological impairments in patients with obsessive compulsive disorder, their healthy siblings and healthy controls: identifying potential endophenotype(s). Psychiatry Res 240:110-117.

Piras F, Piras F, Chiapponi C, Girardi P, Caltagirone C, Spalletta G (2015). Widespread structural brain changes in OCD: a systematic review of voxel-based morphometry studies. Cortex 62:89-108.

Rajender G, Bhatia MS, Kanwal K, Malhotra S, Singh TB, Chaudhary D (2011). Study of neurocognitive endophenotypes in drug-naïve obsessive-compul-sive disorder patients, their first-degree relatives and healthy controls. Acta Psychiatr Scand 124:152-161.

Rao NP, Reddy YC, Kumar KJ, Kandavel T, Chandrashekar CR (2008). Are neu-ropsychological deficits trait markers in OCD? Prog Neuropsychopharmacol Biol Psychiatry 32:1574-1579.

Reitan RM (1955). The relation of the trail making test to organic brain damage. J Consult Psychol 19:393-394.

Riesel A, Endrass T, Kaufmann C, Kathmann N (2011). Overactive errorrelated brain activity as a candidate endophenotype for obsessivecompulsive dis-order: evidence from unaffected first-degree relatives. Am J Psychiatry 168:317-324.

Riesel A, Klawohn J, Grützmann R, Kaufmann C, Heinzel S, Bey K, et al. (2019). Error-related brain activity as a transdiagnostic endophenotype for obses-sive-compulsive disorder, anxiety and substance use disorder. Psychol Med 49:1207-1217.
Rogers RD, Owen AM, Middleton HC, Williams EJ, Pickard JD, Sahakian B J, Robbins TW (1999). Choosing between small, likely rewards and large, unlikely rewards activates inferior and orbital prefrontal cortex. $J$ Neurosci 19:9029-9038.

Segalàs C, Alonso P, Real E, Garcia A, Miñambres A, Labad J, et al. (2010). Memory and strategic processing in first-degree relatives of obsessive com-pulsive patients. Psychol Med 40:2001-2011.

Shallice T (1982). Specific impairments of planning. Philos Trans $R$ Soc Lond B Biol Sci 298:199-209.

Sharma S, Vaish S, Trivedi JK, Dalal PK (2014). Neurocognitive deficits in obses-sive compulsive disorder: a state or trait phenomenon?. J Mental Health Hum Behav 19:78.

Shin NY, Lee TY, Kim E, Kwon JS (2014). Cognitive functioning in obsessive-compulsive disorder: a meta-analysis. Psychol Med 44:1121-1130.

Snyder HR, Kaiser RH, Warren SL, Heller W (2015). Obsessive-compulsive dis-order is associated with broad impairments in executive function: a meta-anal-ysis. Clin Psychol Sci 3:301-330.

Somerville J, Tremont G, Stern RA (2000). The boston qualitative scoring system as a measure of executive functioning in Rey-Osterrieth complex figure perfor-mance. J Clin Exp Neuropsychol 22:613-621.

Schwarz L, Penna S, Novack T (2009). Factors contributing to performance on the rey complex figure test in individuals with traumatic brain injury. Clin Neuropsychol 23:255-267.

Taylor S, Abramowitz JS, McKay D, Cuttler C (2011). Cognitive Approaches to Understanding Obsessive Compulsive and Related disorders. Oxford University Press.

Tezcan D, Tümkaya S (2018). Situation awareness in obsessive compulsive disorder and their relatives: an endophenotype study. Noro Psikiyatr Ars 55:370-375.

Tolin DF, Villavicencio A, Umbach A, Kurtz MM (2011). Neuropsychological func-tioning in hoarding disorder. Psychiatry Res 189:413-418.

Vaghi MM, Hampshire A, Fineberg NA, Kaser M, Brühl AB, Sahakian BJ, et al. (2017). Hypoactivation and dysconnectivity of a frontostriatal circuit during goal-directed planning as an endophenotype for obsessive-compulsive disorder. Biol Psychiatry Cogn Neurosci Neuroimaging 2:655-663.

van Velzen LS, de Wit SJ, Ćurcić-Blake B, Cath DC, de Vries FE, Veltman DJ, et al. (2015). Altered inhibition-related frontolimbic connectivity in obsessive-com-pulsive disorder. Hum Brain Mapp 36:4064-4075.

Viswanath B, Janardhan Reddy YC, Kumar KJ, Kandavel T, Chandrashekar CR (2009). Cognitive endophenotypes in OCD: a study of unaffected siblings of probands with familial OCD. Prog Neuropsychopharmacol Biol Psychiatry 33:610-615.

Watanabe K, Ogino T, Nakano K, Hattori J, Kado Y, Sanada S, Ohtsuka Y (2005). The rey-osterrieth complex figure as a measure of executive function in child-hood. Brain Dev 27:564-569.

Wechsler D (1991). WISC-III: Wechsler Intelligence Scale for Children: Manual. San Antonio, Texas: Psychological Corporation.

Welsh MC, Revilla V, Strongin D, Kepler, M (2000). Towers of hanoi and london: is the nonshared variance due to differences in task administration? Percept Mot Skills 90:562-572.

Whiting P, Rutjes AW, Dinnes J, Reitsma J, Bossuyt P M, Kleijnen J (2004). Development and validation of methods for assessing the quality of diagnostic accuracy studies. Health Technol Assess 8:iii1-iii234.

Williams-Gray CH, Hampshire A, Robbins TW, Owen AM, Barker RA (2007). Catechol O-methyltransferase val158met genotype influences frontoparietal activity during planning in patients with parkinson's disease. $J$ Neurosci 27:4832-4838.

Zhang J, Yang X, Yang Q (2015a). Neuropsychological dysfunction in adults with early-onset obsessive-compulsive disorder: the search for a cognitive endo-phenotype. Braz J Psychiatry 37:126-132.

Zhang L, Dong Y, Ji Y, Zhu C, Yu F, Ma H, Wang K (2015b). Dissociation of decision making under ambiguity and decision making under risk: a neuro-cognitive endophenotype candidate for obsessive-compulsive disorder. Prog Neuropsychopharmacol Biol Psychiatry 57:60-68. 